\title{
Stratégie d'exploitation des nappes de l'Albien et du Néocomien en région parisienne
}

\author{
par Bernard Grenet \\ de l'Agence de l'Eau Seine-Normandie \\ par Hugues Haeffner \\ de la Société Lyonnaise des Eaux \\ par Annick Samson \\ de l'Agence de l'Eau Seine-Normandie
}

par Pierre Suzanne

de la Société Lyonnaise des Eaux

Beaucoup de nappes profondes sont captives sous une couverture peu perméable et, de ce fait, sont protégées des pollutions de surface mais mal alimentées en eau.

Leur exploitation doit tenir compte de ces caractéristiques. En conséquence:

- on ne peut les exploiter de façon permanente qu'à des débits faibles;

- il faut privilégier l'utilisation temporaire de leurs réserves souvent considérables;

- enfin, il faut tenir compte de leurs relations par drainance avec les aquifères qui les encadrent.

Ce sont ces trois points que veut illustrer cet article à travers l'exemple des nappes de l'Albien et du Néocomien du bassin de Paris.

\section{CARACTÉRISTIQUES STRUCTU- RALES ET HYDROGÉOLOGIQUES DES AQUIFERES}

La structure générale du bassin parisien est bien connue : c'est une vaste cuvette, formée d'une superposition «à la manière d'une pile d'assiettes » de couches sédimentaires déposées au cours des ères secondaire et tertiaire, de natures lithologiques variées et qui reposent sur un socle ancien affleurant sur leur pourtour: Bretagne, Vosges, Ardennes et Massif Central.

La nappe de l'Albien est située dans la formation géologique du même nom (fïn du crétacé inférieur) et est constituée de niveaux sableux alternant avec des passées argileuses. Elle adopte la même structure en cuvette que l'ensemble des couches du bassin. Son toit est situé, en région parisienne, à une profondeur comprise entre 500 et 800 mètres.

Elle est présente sous la majeure partie du bassin parisien (fig. I) et sa superficie d'environ $75000 \mathrm{~km}^{2}$ en fait l'aquifère le plus étendu de France.

Situé à plusieurs dizaines de mètres sous l'Albien, l'aquifère du Néocomien au sens large n'est pratiquement connu que par les mesures géophysiques et les forages pétroliers. En effet, à l'heure actuelle, un seul forage d'eau y fait appel (un deuxième vient cependant d'être autorisé).

La nappe du Néocomien s.l. est développée dans les formations continentales (faciès Wealdien) du Néocomien et du

After a short reminder on the structural and hydrogeological characteristics of the aquifers, the authors will present the strategic stakes of the exploitation of the confined aquifers in Graeter Paris. A stidy using the mathematical model of the Chalk-Albian-Neocomian system laid the foundations to assess the exchanges between the different aquiferous systems and to characterize the interdependence linking them. The results obtaned enable reseachers to propose a rational exploitation of the aforesaid aquifers. 


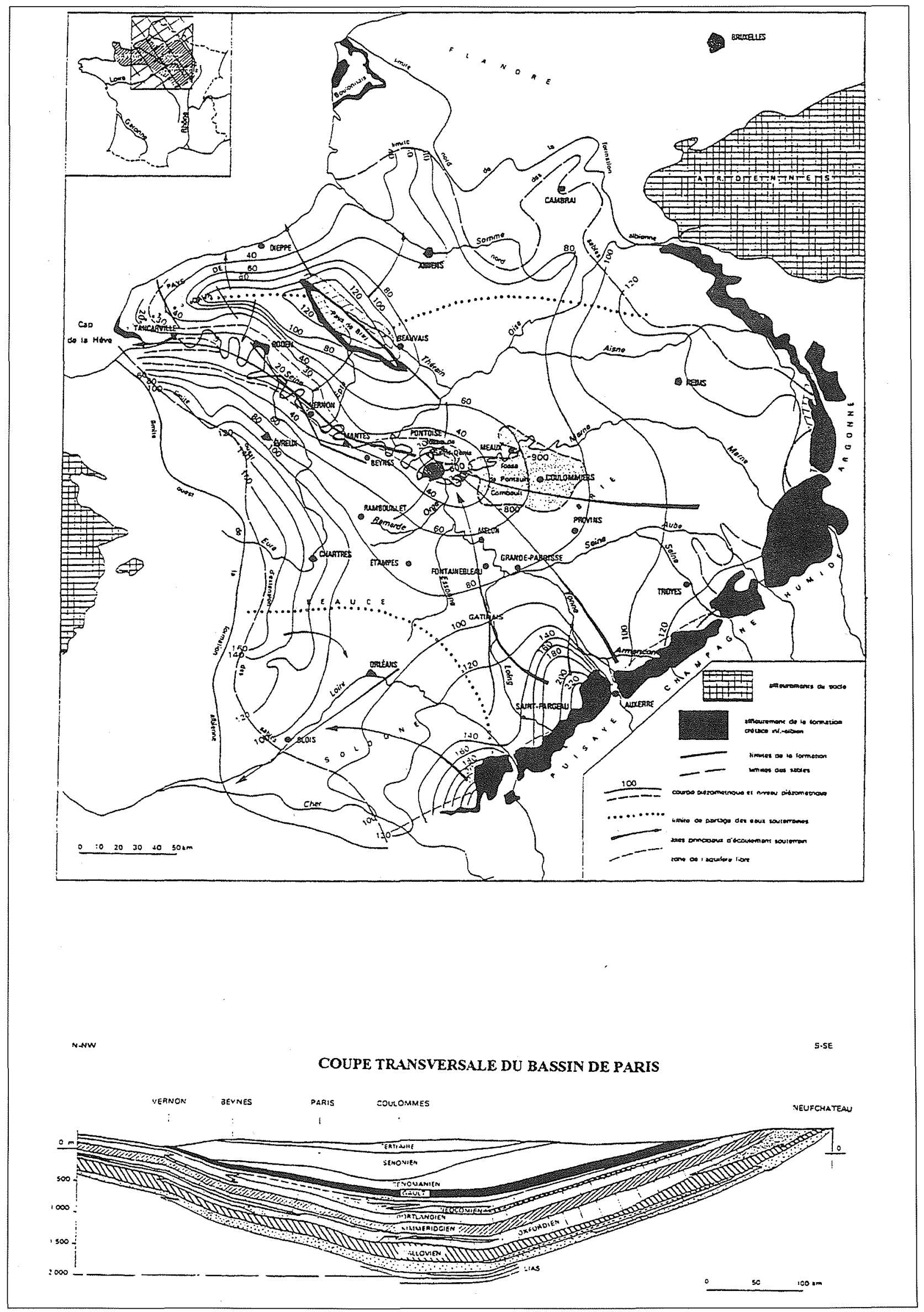

1. Piézométrie de la nappe de l'Albien. 
Barrémien inférieur représentées par une succession de couches sableuses et gréseuses alternant avec des argiles bariolées. Elle est séparée de l'Albien par les niveaux argilosableux du Barrémien supérieur et de l'Aptien.

Les formations ne sont affleurantes que sur la bordure orientale et dans la «boutonnière » du pays de Bray. Leur extension vers l'ouest est beaucoup plus réduite que celle de l'Albien. En Ile de France, la profondeur du toit varie entre 600 et 1000 mètres dans la fosse de la Brie.

Les argiles du Gault, qui constituent le sommet de la formation albienne, recouvrent pratiquement partout l'aquifère et le maintiennent en charge sur la quasi-totalité de sa superficie, sauf à la périphérie où les sables affleurent, ce qui permet une certaine alimentation par l'infiltration des précipitations.

La piézométrie actuelle de la nappe de l'Albien montre un écoulement général de l'est vers l'ouest avec un sillon central marqué suivant l'axe de la Seine.

Au siècle dernier les eaux étaient partout jaillissantes en Ile de France à plusieurs dizaines de mètres de hauteur ce qui avait fait supposer une ressource considérable. Aujourd'hui, après 60 ans d'une exploitation relativement modérée $\left(0,6\right.$ à $0,8 \mathrm{~m}^{3} / \mathrm{s}$ depuis 1930$)$ le niveau piézométrique est légèrement au-dessous du sol sauf en certains points de l'ouest parisien où l'on observe encore un léger artésianisme.

La nappe de l'Albien est encadrée par deux autres niveaux aquifères:

- la nappe de la craie sus-jacente, dont elle est séparée par les argiles du Gault et surtout par la base même de la craie, peu perméable et épaisse,

- la nappe du Néocomien, au-dessous, dont le niveau piézométrique est identique à celui de l'Albien près des affleurements mais lui est nettement supérieur en lle de France en raison de l'exploitation de la nappe albienne.

Les séparations entre nappes ne sont cependant pas rigoureusement étanches et, sous l'effet de l'exploitation, la nappe albienne peut recevoir, par drainance, des volumes d'eau importants de ces deux aquifères encaissants.

Inversement, l'exploitation intensive de ces deux aquifères, notamment le Néocomien, pourrait soutirer à la nappe de l'Albien des volumes non négligeables.

\section{ASPECTS STRATÉGIQUES DE LA NAPPE DE L'ALBIEN}

C'est en 1841, après 7 ans de travaux, que l'eau a jailli du premier forage à la nappe de l'Albien dans la cour de l'abattoir de Grenelle à Paris, laissant envisager une ressource considérable et gratuite.

L'exploitation est passée ensuite par diverses étapes (fig. 2): jusqu'en 1930 une quinzaine de forages seulement furent réalisés. En 1930 l'adaptation aux forages d'eau de la technique pétrolière «rotary » (forage en rotation à la boue) provoqua une explosion du nombre de puits: une trentaine d'ouvrages furent réalisés en 5 ans et les prélèvements annuels atteignirent $34 \mathrm{Mm}^{3}$, concentrés en région parisienne. La baisse piézométrique devint alors catastrophique plus de 80 mètres de chute par rapport à 1841

En 1935, pour juguler cette baisse, une législation spéciale fut mise en place (décret-loi de 1935). Elle soumettait à autorisation préalable tous les forages de plus de 80 mètres de profondeur $\left({ }^{1}\right)$ : le service des Mines, chargé de son application, n'accordera alors de nouvelles autorisations qu'en remplacement de «droits d'eau » abandonnés.

1. En Ile-de-France seulement.

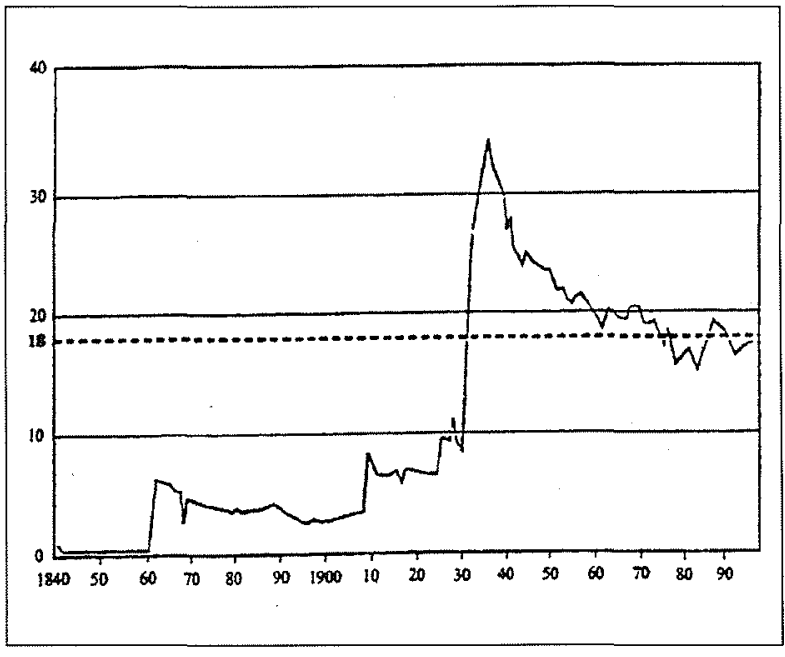

2. Nappe de l'Albien. Historique des volumes exploités (en millions de $\mathrm{m}^{3} / \mathrm{an}$ ).

En 1979 un groupe de travail, sous l'égide du service des mines, fixa le volume annuel d'exploitation à ne pas dépasser en Ile-de-France à $18 \mathbf{M m}^{3}$ et préconisa la réservation des eaux de l'Albien à l'usage d'eau potable. Jusqu'en 1984 les prélèvements oscilleront autour de $15 \mathrm{Mm}^{3} /$ an et les niveaux remonteront. Mais depuis cette date, une certaine reprise des prélèvements (forages AEP de Neuilly-sur-Seine notamment) a provoqué une nouvelle baisse.

La loi sur l'eau de 1993 ayant abrogé le décret-loi de 1935, mais maintenu le régime d'autorisation préalable pour les forages de plus de 80 mètres quel que soit leur débit, dans les zones où il s'appliquait, la nappe est théoriquement protégée, du moins en Ile-de-France.

Mais depuis quelques années, on observe deux tendances inquiétantes :

- la création de nouveaux forages aux limites de l'Ilede-France, dans chacun desquels il est possible de prélever, cette fois sans autorisation préalable, jusqu'à $80 \mathrm{~m}^{3} /$ heure, soit $700000 \mathrm{~m}^{3}$ par an.

- des demandes d'autorisation de forage à la nappe du Néocomien, considérée comme une ressource indépendante de l'Albien.

Cependant, les particularités géométriques et géologiques exposées ci-dessus, ainsi que l'examen des conséquences catastrophiques de l'exploitation passée montrent à l'évidence que cette nappe ne peut pas être exploitée comme un aquifère classique.

Elle possède en effet des caractéristiques originales:

- des réserves géologiques considérables (plusieurs centaines de milliards de $\mathrm{m}^{3}$ )

- une alimentation naturelle très faible vis-à-vis de ces réserves: on l'a comparée à une grande retenue de barrage qui ne serait alimentée que par un petit ruisseau ;

- une grande «nervosité » liée à son caractère captif (diffusivité importante) : toute perturbation, pompage ou injection, s'y propage rapidement et loin ;

- une température des eaux élevée : jusqu'à $35^{\circ} \mathrm{C}$ dans l'est de l'Ile de France:

- une protection totale vis-à-vis des pollutions de surface

\section{En conséquence...}

... la nappe de l'Albien doit être considérée comme une réserve stratégique primordiale pour la région parisienne en cas d'indisponibilité durable des ressources classiques.

Les calculs exposés dans la suite de cet article montreront que le Néocomien n'est pas une ressource distincte de l'Albien et que sa mise en exploitation ne permet pas 
d'accrôttre, sinon à court terme, les volumes totaux exploitables.

Une exploitation intensive de cette nappe peut donc, par drainance, abaisser le niveau piézométrique de l'Albien et rendre inutilisables (ou du moins inexploitables économiquement) les forages actuels.

\section{MODÉLISATION DU COMPLEXE AQUIFÈRE ALBIEN-NÉOCOMIEN}

Dans le cas de systèmes aquiferes complexes, tels ceux de l'Albien et du Néocomien, l'utilisation de modèles hydrodynamiques est le seul moyen permettant de bien quantifier les mouvements de l'eau et en particulier d'évaluer l'impact d'une politique d'exploitation.

A partir des données actuellement disponibles, un modèle des systèmes aquifères de l'Albien et du Néocomien ainsi que de l'aquifère de la Craie a donc été réalisé en 1995 en vue d'obtenir des estimations fiables des phénomènes hydrodynamiques mis en jeu.

\section{Caractéristiques du modèle:}

Afin de prendre en compte correctement l'alimentation des nappes par les affleurements en bordure, ainsi que les effets de drainance, l'ensemble du système Craie-Albien-Néocomien a été modélisé.
Ce modèle s'étend sur l'ensemble de la zone du Bassin Parisien où l'une des trois couches aquifères est présente, soit environ un carré de $300 \mathrm{~km}$ de côté. Au total 2929 mailles carrées de $10 \mathrm{~km}$ de côté forment la structure du modèle.

L'ensemble des caractéristiques géométriques des aquifères (étendue, profondeur, épaisseur), la géométrie des principaux cours d'eau, ainsi que la position des forages à l'Albien et au Néocomien ont été prises en compte.

Les caractéristiques hydrodynamiques des aquifères (emmagasinement et transmissivité) ont été obtenues à partir de nombreuses informations bibliographiques et archives. Les coefficients de drainance entre les aquifères ont été ajustés lors du calage du modèle et sont discutés plus loin.

La piézométrie de la nappe de la Craie a été facilement reconstituée en considérant l'équilibre dynamique entre les précipitations et le drainage par les principaux cours d'eau représentés par des potentiels imposés. Cette piézométrie est très stable et permet de bien rendre compte du potentiel imposé à la nappe de l'Albien par la nappe de la craie.

Pour la nappe de I'Albien, les affleurements de Champagne sont considérés comme des limites à potentiel imposé. Ceci est justifié étant donné que les précipitations totales sur ces affleurements sont très nettement supérieures aux débits qui peuvent s'écouler dans la nappe de l'Albien, même en cas de surexploitation. La nappe Albienne est en contact par drainance avec les nappes de la craie et du Néocomien. Le calage des coefficients de drainance a permis de reproduire la piézométrie actuelle de façon satisfaisante (fig. 3). Les coefficients retenus entre la craie et l'Albien sont de

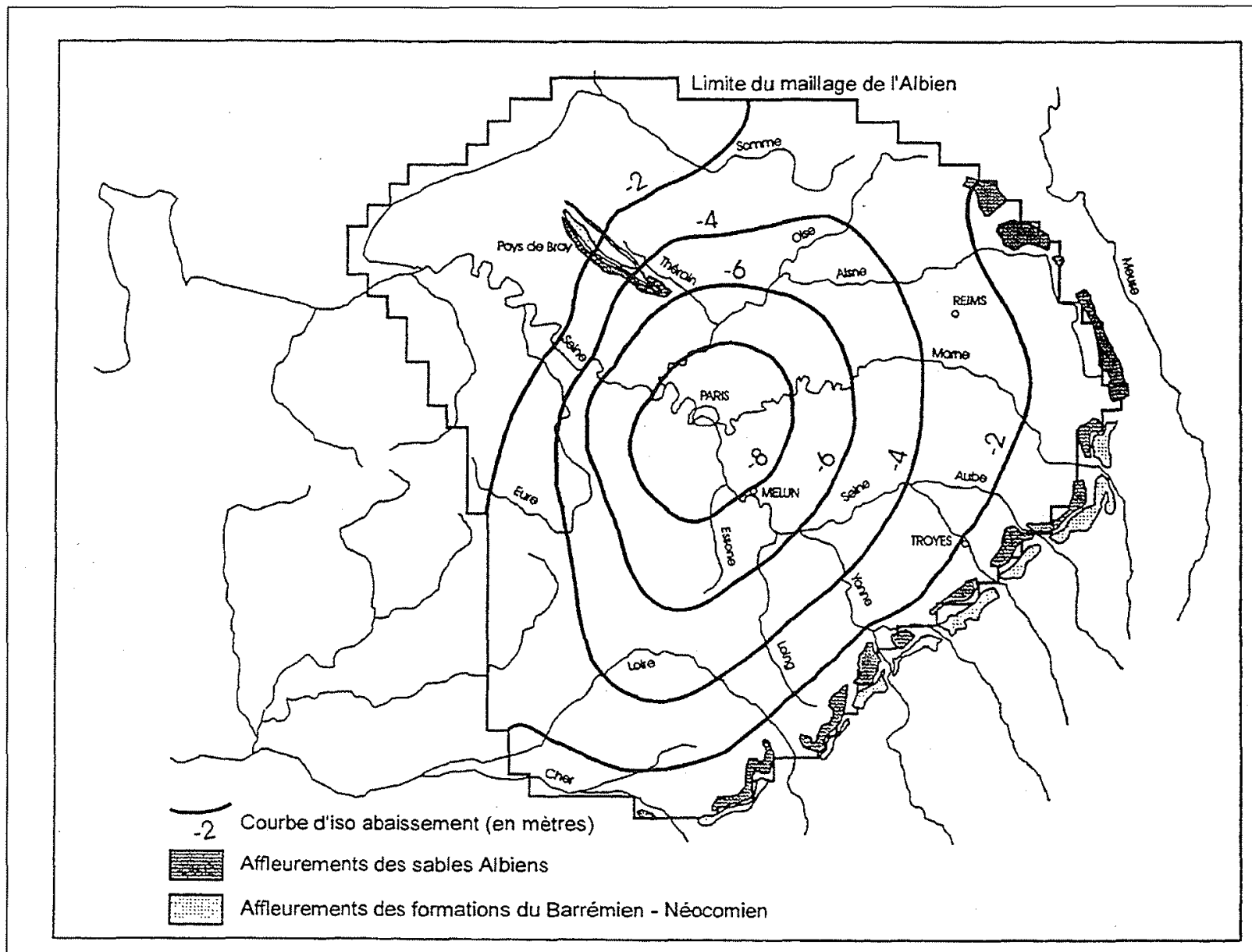

3. Piézométrie actuelle simulée de la nappe de l'Albien. 
2.1 $10^{-6} \mathrm{~h}^{-1}$ dans l'axe de la basse Seine et de $8.10^{-8} \mathrm{~h}^{-1}$ sur le reste du bassin. Cette dernière valeur correspond à une couche de $45 \mathrm{~m}$ d'épaisseur ayant une perméabilité faible de $10^{-9} \mathrm{~m} / \mathrm{s}$. Des valeurs différentes ne permettent pas d'obtenir une piézométrie correcte en région parisienne à moins de changer l'ordre de grandeur des perméabilités horizontales de la nappe ce qui serait en contradiction avec de nombreuses valeurs expérimentales.

Pour la nappe du Néocomien, les affleurements de la région de Champagne ont été également représentés par des potentiels imposés. Les transmissivités horizontales, conformément aux données existantes, ont été prises égales ì $2 \cdot 10^{-3} \mathrm{~m}^{2} / \mathrm{s}$ au sud de la région parisienne et à $0.5 \cdot 10^{-3} \mathrm{~m}^{2} / \mathrm{s}$ sur le reste du bassin. L'exutoire de la nappe est constitué par la drainance vers l'aquifère Albien dans la région parisienne et l'axe de la basse Seine. Pour obtenir une piézométrie correcte au niveau de l'Ile de France, on constate la nécessité d'un coefficient de dramance compris entre 6 et $9.10^{-8} \mathrm{~h}^{-1}$. Ceci correspond également à des perméabilités de $10^{-9} \mathrm{~m} / \mathrm{s}$ pour les formations peu perméables séparant l'Albien et le Néocomien.

\section{Exploitation du modèle}

Le modèle mathématique représentatif des aquifères Albien et Néocomien a été utilisé pour évaluer quantitativement les conséquences de deux scénarios d'exploitation. Par rapport à la situation actuelle, où l'exploitation de la nappe de
l'Albien est stabilisée autour de 18 millions de $\mathrm{m}^{3} / \mathrm{an}$ (soit $2060 \mathrm{~m}^{3} / \mathrm{h}$ ), des pompages supplémentaires de $600 \mathrm{~m}^{3} / \mathrm{h}$ répartis sur plusieurs forages en région parisienne ont été simulés soit dans l'Albien soit dans le Néocomien. Il faut rappeler qu'une autorisation de prélèvement de $300 \mathrm{~m}^{3} / \mathrm{h}$ dans le Néocomien a été accordée récemment.

Les simulations ont été réalisées en régime permanent. Les résultats obtenus sont donc les conséquences «à l'équilibre » d'un schéma d'exploitation des systèmes aquifères considérés. Dans les faits, on observe que la piézométrie de l'Albien se stabilise au bout de 10 à 20 ans pour des périodes où les prélèvements sont à peu près constants.

Les résultats des simulations mettent en évidence des échanges importants par drainance entre les aquifères:

Pour un prélèvement dans I'Albien :

- $78 \%$ de l'eau provient des échanges avec la Craie.

- $18 \%$ provient des apports par les limites (Champagne, pays de Bray),

- $3 \%$ provient du Néocomien.

Pour un prélèvement dans le Néocomien :

- $89 \%$ de l'eau provient de la nappe de l'Albien,

- $11 \%$ provient des apports par les limites du Néocomien.

On constate ainsi que la nappe du Néocomien est principalement alimentée par drainance et que tout pompage dans le Néocomien a une répercussion forte sur l'Albien.

Lors des simulations de pompage dans le Néocomien on observe qu'un rabattement significatif de la nappe de l'Albien se produit (voir tableau ci-après et fig. 4).

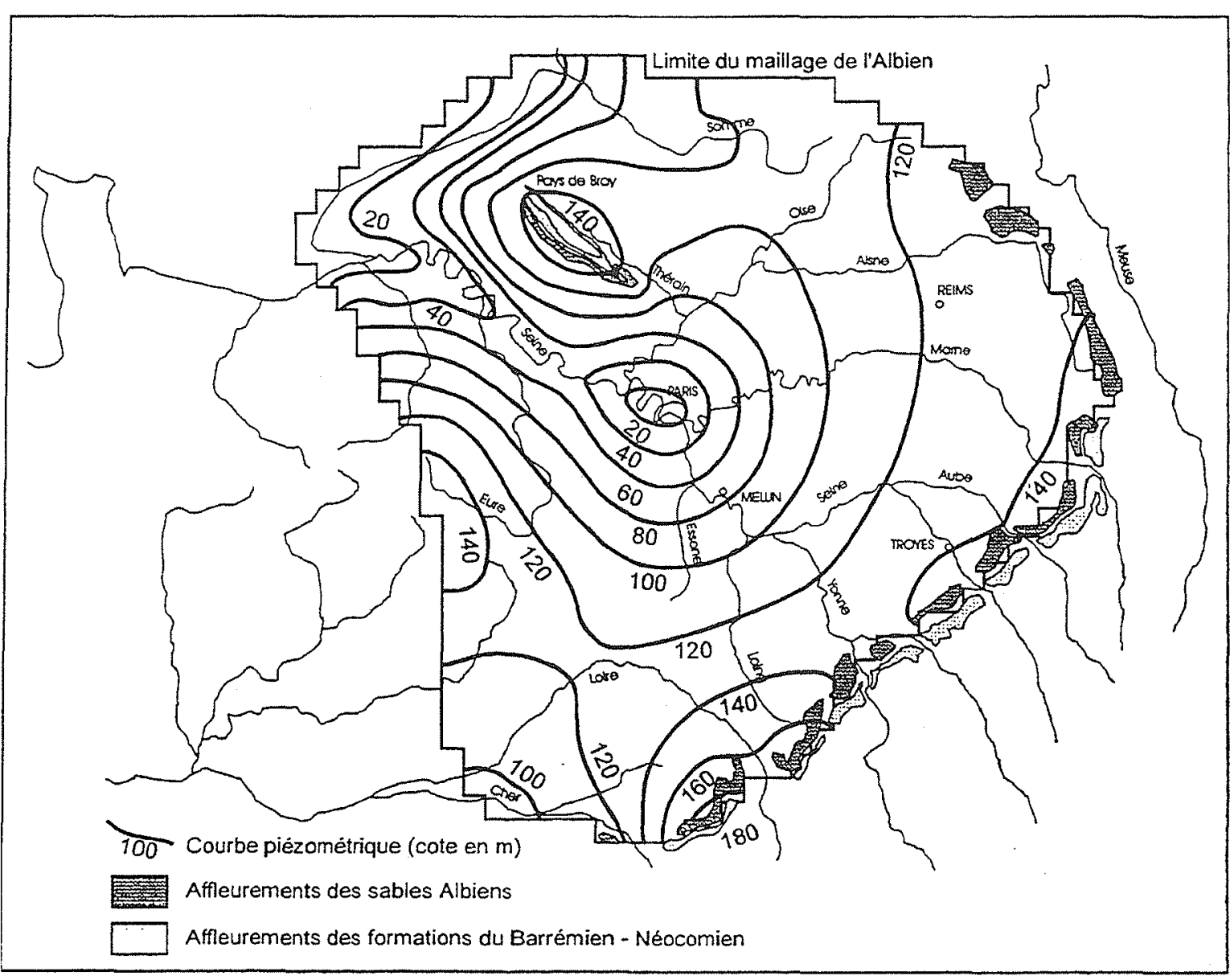

4. Rabattement de la nappe de l'Albien lors d'un pompage de $600 \mathrm{~m}^{3} / \mathrm{h}$ dans la nappe du Néocomien. 


\begin{tabular}{|c|c|c|}
\hline $\begin{array}{c}\text { Pompage } \\
\text { supplémentaire }\end{array}$ & $\begin{array}{c}600 \mathrm{~m}^{3 / \mathrm{h}} \\
\text { dans le Néocomien }\end{array}$ & $\begin{array}{c}600 \mathrm{~m}^{3} / \mathrm{h} \\
\text { dans l'Albien }\end{array}$ \\
\hline $\begin{array}{c}\text { Rabattement de la nappe de l'Albien } \\
\text { en région parisienne }\end{array}$ & 8 à 9 mètres \\
\hline $\begin{array}{c}\text { Rabattement de la nappe } \\
\text { du Néocomien en région parisienne }\end{array}$ & 35 mètres & 15 à mètres \\
\hline
\end{tabular}

Ces résultats indiquent clairement que la seule limitation des pompages dans l'Albien n'est pas suffisante pour préserver son potentiel hydraulique mais qu'il convient de limiter également les pompages dans l'aquifère du Néocomien.

\section{UN EXEMPLE D'EXPLOITATION RATIONNELLE DE LA NAPPE ALBIENNE}

Pour tenter de résumer les diverses études réalisées sur la nappe de l'Albien, et notamment les résultats des modèles mathématiques réalisés en 1974 et 1995 , on peut dire qu'elles ont mis en évidence deux points essentiels :

- on ne peut l'exploiter, de façon permanente, qu'à des débits faibles si l'on veut conserver des niveaux piézométriques acceptables. Les 18 millions de $\mathrm{m}^{3}$ par an fixés comme limite par le groupe de travail en 1979 ne sont donc qu'une goutte d'eau dans l'océan des besoins de l'agglomération parisienne, environ 50 fois plus élevés :

- en corollaire, il serait possible de l'exploiter temporairement à très fort débit et de laisser se reconstituer les réserves par la suite, à condition, bien sûr, de disposer des ouvrages d'exploitation ad hoc.

Le respect de ces deux caractéristiques permet d'imaginer une exploitation de l'Albien axée sur l'alimentation d'un réseau de sécurité ultime permettant de faire face soit à une pollution de longue durée de la totalité des ressources, soit à une indisponibilité prolongée d'énergie.

En dehors de ces périodes de crise ce réseau serait utilisé pour alimenter une série de fontaines décoratives fournissant une eau «de bouche » de qualité exceptionnelle pouvant concurrencer partiellement les eaux embouteillées.

Les principales caractéristiques pourraient être les suivantes:

- un réseau entièrement distinct du réseau d'alimentation en eau potable habituel...

- ...alimenté par un nombre important de forages répartis de façon homogène...

- ...exploités en temps normal à des débits unitaires faibles pour faire fonctionner une série de fontaines décoratives pouvant fournir de l'eau de bouche de qualité...

- ...mais pourvus de systèmes d'alimentation autonomes permettant, en temps de crise, de faire fonctionner des pompes à fort débit pour l'approvisionnement de camionsciternes, par exemple.

\section{ESTIMATION SOMMAIRE POUR LA VILLE DE PARIS}

Données de dimensionnement :

* Besoins incompressibles de crise :

$35 \mathrm{l} / \mathrm{hab}$./jour, soit, pour 2100000 habitants, $73500 \mathrm{~m}^{3} / \mathrm{j}$.

* Pour satisfaire ces besoins il est nécessaire de réaliser 20 forages à la nappe de l'Albien pouvant débiter chacun $150 \mathrm{~m}^{3} / \mathrm{h}$, soit $72000 \mathrm{~m}^{3} / \mathrm{j}$.

* Chaque forage alimente un réseau indépendant constitué de conduites disposées « en étoile » à partir du forage et fournissant l'eau à 12 fontaines.

On obtient ainsi un ensemble de 240 fontaines régulièrement disposées de façon qu'aucun parisien ne soit distant de plus de 350 mètres de l'une d'elles.

* Le débit de chaque fontaine, de quelques dizaines de litres/minute en temps normal (réseau d'eau de bouche), doit pouvoir être porté, en temps de crise, à environ $300 \mathrm{~m}^{3} /$ jour $\left(12.5 \mathrm{~m}^{3} / \mathrm{h}\right)$ pour alimenter un réservoir de $500 \mathrm{~m}^{3}$ et des citernes auxiliaires munies de plusieurs robinets (atténuation des files d'attente)

Estimation sommaire des coûts d'investissement :

Pour un réseau :

- Conduites : $8000 \mathrm{ml}$ en $\phi 100 \mathrm{~mm}$ (zone urbaine dense)

- Forage à l'Albien $(700 \mathrm{~m})$ et réservoir de $500 \mathrm{~m}^{3}$

- Fontaines : 12 fontaines à $50000 \mathrm{~F}$ et citernes auxiliaires

Soit, pour 20 réseaux : 500 MF (238 F par habitant)

A titre de comparaison...

- le réseau de fontaines de la ville de Zürich peut être évalué, en Francs 1995, à 120 MF pour 420000 habitants, soit 286 F/hab.,

- la réfection d'une seule des trois usines de traitement d'eau potable de la ville de Paris revient à plus de 600 MF. 
En annexe est présenté un essai d'estimation des coûts d'investissement d'un tel réseau de sécurité appliqué à la ville de Paris. Le coût total, estimé à 500 MF, est acceptable si on le compare, par exemple, à la rénovation d'une seule des trois usines de traitement d'eau superficielle qui alimentent la capitale: plus de $600 \mathrm{MF}$.

Le coût pour un habitant (2) reste également modéré : $238 \mathrm{~F}$, soit environ le tiers de sa facture d'eau d'une année.

Cet exemple appliqué à la seule ville de Paris pourrait être étendu à l'ensemble de l'agglomération parisienne moyennant une surexploitation temporaire plus importante de la nappe que les réserves géologiques considérables de celle-ci permettent d'envisager sans crainte.

\section{$\mathrm{V}$ 䁗 CONCLUSION}

Les nappes profondes de l'Albien et du Néocomien du bassin parisien, en raison de leurs réserves en eau considérables et de leur protection totale vis à vis des pollutions de surface, doivent être considérées comme aquifères d'intérêt stratégique.

Il est donc nécessaire de les protéger, tant quantitativement que qualitativement, de façon globale, c'est-à-dire en prenant en compte les relations hydrauliques étroites qui existent entre elles.

On ne peut prélever en permanence dans ces deux nappes que des volumes relativement modestes, surtout si on les compare aux besoins de la région parisienne. Il est cependant possible d'imaginer un mode d'exploitation qui assure à la fois une fourniture permanente d'eau «de bouche» de qualité et une alimentation minimale en cas de crise majeure.

\section{Références}

[1] Berger G. - La nappe de l'Albien en lle de France, synthèse et actualisation des données, perspectives d'exploitation. Rapport B.R.G.M. 78 SGN 697 BDP, décembre 1978 (pour l'Agence de l'Eau Seine-Normandie et le Ministère de l'Industrie).
[2] Berger G. et Blomere P. - Nappe de l'Albien : étude de l'évolution piézométrique de 1981 à 1993, incidence des prélèvements. Rapport B.R.G.M. 94 DSGR 001 IDF, mars 1994. (pour le Ministère de l'Industrie - DRIRE d'lle de France)

[3] BUR GE.AP. - Modèle mathématique pour l'étude et la gestion de la nappe de l'Albien dans la région parisienne. Janvier 1975 (pour la Compagnie Générale des Eaux).

[4] Camart R. et Moussie B. - Compte rendu provisoire des résultats de pompages d'essais sur le forage géothermique du C.E.A. de Bruyères-le-Châtel (91). Rapport B.R.G.M. 81 IDF 036, août 1981

[5] FREY Ch. et al. - Etude hydrogéologique de la nappe de l'Albien en région Ile de France. Rapport B.R.G.M. 81 SGN 800 IDF, novembre 1981 (pour la Région Ile de France et le Ministère de l'Industrie).

[6] GEOTHERMA. - Synthèse des données acquises sur le réservoir du Néocomien à l'aide des forages géothermiques récents. Rapport provisoire GEOTHERMA, mai 1986.

[7] GEOTHERMA. - Etude d'inventaire thermique des nappes aquilères de 100 à $1000 \mathrm{~m}$ en région Ile de France, fascicule $\mathrm{n}^{\circ} 1$, Inventaire des ressources en eaux souterraines. Janvier 1984 (pour l'IAURIF).

[8] Lauveruat J. (1967). - Contribution à l'étude géologique el hydrogéologique de l'Albien dans le centre du bassin de Paris. Thèse de $3 \mathrm{e}$ cycle, Paris

[9] Maget. et al. - Potentiel géothermique « basse température » en France. Rapport B.R.G.M. 83 SGN 375 SPG (pour la Commission des Communautés Européennes et le B.R.G.M.).

[10] Mission déléguée de Bassin Seine-Normandie, Agence FinanCiere de Bassin Seine-Normandie. - Les bassins de la Seine et des cours d'eau normands, tome 1 : ressources d'eau et données hydrologiques, fascicule 4 : eaux souterraines 2e trimestre 1974.

[11] SERVice interdépartemental de L'INDUSTRIE ET DES Mines D'ILE DE FRANCE. - Définition d'une gestion rationnelle de la nappe albienne. Février 1979.

[12] Verrjer T. - Les réseaux de fontaines publiques et la sécurité de l'alimentation en région parisienne : principes de conception, coûts, fréquentation probable. Rapport BETURE. mars 1980 (pour l'Agence de l'Eau Seine-Normandie). 